\title{
Influence of the Particle Concentration and Marangoni Flow on the Formation of Cellulose Nanocrystal Films
}

\author{
Alican Gençer, Christina Schütz, ${ }^{(0)}$ and Wim Thielemans* ${ }^{*}$ \\ Renewable Materials and Nanotechnology Research Group, Department of Chemical Engineering, KU Leuven, Campus Kulak \\ Kortrijk, Etienne Sabbelaan 53, 8500 Kortrijk, Belgium
}

\section{Supporting Information}

ABSTRACT: Cellulose nanocrystals (CNCs), ribbonlike crystalline nanoparticles, are a biobased material that can be a great alternative to obtaining films with tunable optical properties. Iridescent and light-diffracting films can be readily obtained via the drying of a suspension of these cellulose nanocrystals. The characteristics of the particle deposition process together with the self-assembly in the precluding suspension has a direct effect on the optical properties of the obtained films. Particle deposition onto a substrate is affected by the flow dynamics inside sessile droplets and usually yields a ringshaped deposition pattern commonly referred to as the coffee-ring effect. We set out to measure and describe the drying kinetics under different conditions. We found that the Marangoni flow inside the droplet was too small to counteract the capillary flow that deposits CNCs at the edges, resulting in the coffee-ring effect, irrespective of the atmospheric humidity. By varying the amount of ethanol in the atmosphere, we were able to find a balance between (1) colloidal stability in the droplet, which is reduced by ethanol diffusion into the droplet, and (2) increasing Marangoni flow relative to capillary flow inside the droplet by changing the droplet surface tension. We could thus make iridescent films with a uniform thickness.

\section{INTRODUCTION}

Multifunctional films can be produced via deposition of a suspension onto a substrate followed by drying of the sessile droplet. ${ }^{1}$ However, the drying of sessile droplets containing colloidal particles commonly leaves a ring-shaped deposit as the solute particles have a tendency to flow toward the edge of the droplet. ${ }^{2}$ The accumulation of particles at the edge of the droplet is caused by the evaporation rate being higher at the edge generating a capillary flow that induces a net transport of particles toward the edge. A recent study showed that the concentration of rodlike cellulose nanocrystals at the edge of an evaporating droplet, due to a buildup of nanocrystals at the outer edge of the droplet, can be high enough to induce gel formation. ${ }^{3}$ Colloidal dispersions of cellulose nanocrystals are also known to self-assemble in aqueous suspensions into chiral nematic structures well above their gel point, which may also affect the deposition pattern. ${ }^{4-6}$ It is thus crucial to understand what happens during the deposition of colloidal particles to be able to exert control over the achieved deposition pattern and the final optical properties of the dried film. Precise control of drop casting is also important to understanding deposition effects during the fabrication of films with different optical properties via techniques such as inkjet printing. ${ }^{7-10}$

Neat CNC films tend to be quite brittle in nature, so they are sometimes combined with other molecules to make them more flexible. ${ }^{11}$ Several studies showed increased flexibility of CNC

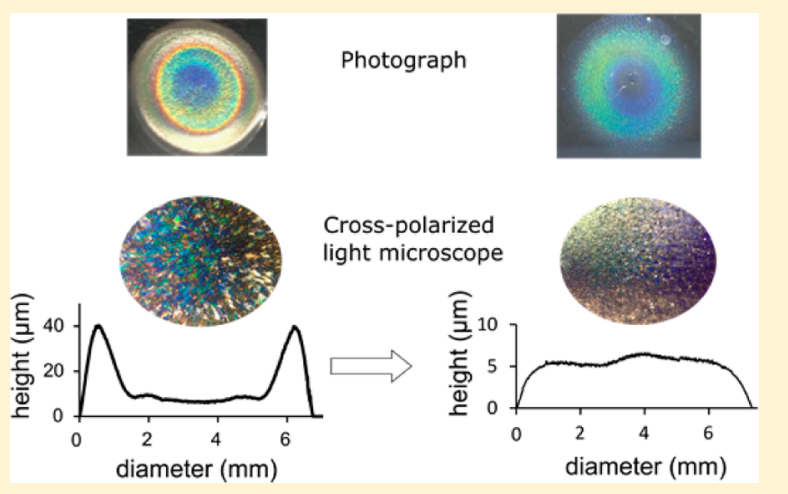

films could be achieved by codeposition with polymers such as poly(ethylene glycol $)^{12}$ and poly(vinyl alcohol) $)^{13}$ or with zwitterionic surfactants. $^{14}$

Previously, suspensions of cellulose nanocrystals in water and polar organic solvents have been evaporated under ambient conditions and under vacuum to determine the effect of the evaporation rate on the cast films. ${ }^{15}$ It was concluded that the colloidal stability of the suspensions in polar organic solvents was not feasible without any water present. Contrary to this work, a dispersion of cellulose nanocrystals was achieved in polar organic solvents via a neutralized form of CNCs (CNC-X, $\mathrm{X}=\mathrm{Li}, \mathrm{Na}, \mathrm{K}$, etc.), but in this work, the authors did not mention verifying the water content of the used solvents or drying them extensively. ${ }^{16}$ Another study carried out on suspensions of inorganic nanoparticles of $\mathrm{TiO}_{2}, \mathrm{Fe}_{2} \mathrm{O}_{3}$, $\mathrm{SrCO}_{3}$, and $\mathrm{Co}_{3} \mathrm{O}_{4}$ achieved control over deposition by investigating the shape changes of deposits by restricting the evaporation rate and by combining high- and low-boiling-point solvents. ${ }^{17}$ Evaporation-induced self-assembly of oxidized cellulose nanocrystals in a shrinking droplet was reported to control the morphology of microbeads immersed in a binary toluene-ethanol mixture. ${ }^{18}$ Similarly, the relation between the

Received: October 12, 2016

Revised: December 6, 2016

Published: December 7, 2016 
(b)

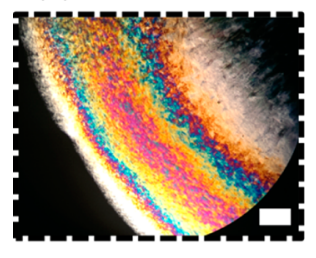

(a)

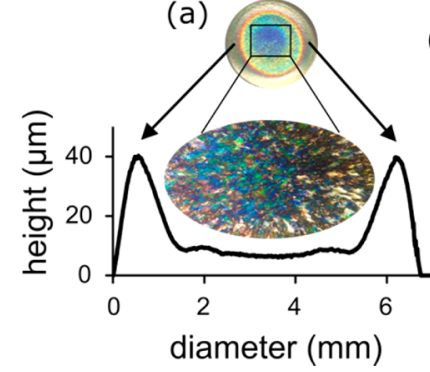

(c)

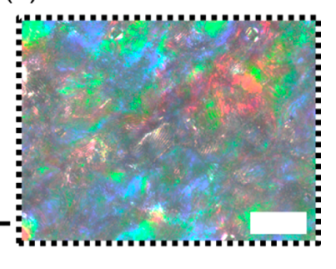

Figure 1. Film obtained by drying a $3.7 \mathrm{wt} \%$ cellulose nanocrystal suspension at $40 \% \mathrm{RH}$ as viewed (a) from the top by the naked eye, (b) at the edge of the film under a cross-polarized light microscope (scale bar $200 \mu \mathrm{m}$ ), and (c) in the center of the film under a cross-polarized light microscope (scale bar $50 \mu \mathrm{m})$ and height profile of the film as a function of diameter.

fibril shape and the self-organizing capacity was investigated by comparing the results of evaporation-induced self-assembly in coffee rings and spray-dried microparticles for rodlike and semiflexible tunicin nanofibers. ${ }^{19}$ The semiflexible tunicin nanofibers did not show a phase transition with evaporation, whereas rodlike cellulose nanocrystals self-assembled into nematic fragments.

In this work, the drying process of cellulose nanocrystal suspensions was studied under different environments to obtain iridescent films with a uniform thickness. The deposition pattern was controlled using the relative magnitude of Marangoni flow inside the droplet by manipulating the droplet environment. Achieving a uniform thickness also avoided thin film interference by making sure that colors arose only from uniform film iridescence and were homogeneous throughout the film.

To study the conditions needed to change the ring-shaped deposit to a deposit with uniform thickness, films were prepared from suspensions with varying concentrations of cellulose nanocrystals and dried under ambient, humiditycontrolled and ethanol atmospheres to alter the internal Marangoni flow. The height profiles of the prepared films were correlated with crossed-polarized light microscope images of those films. An analytical expression was formulated to understand the mass deposition rate during droplet drying and the concentration changes in the suspension for the evaporating droplet. The colloidal stability of the suspensions was also characterized by zeta potential and rheological measurements. The insights we developed were then used to prepare constantthickness films with uniform film iridescence.

\section{EXPERIMENTAL SECTION}

Materials. Cotton wool, calcium chloride (96\%, granular), and isopropanol (99.5\%, HPLC grade) were purchased from Carl Roth GmbH \& Co. KG. Sulfuric acid (95\%, RECTAPUR) and ethanol (96\%, Analar Normapur) were purchased from VWR International. Potassium bromide, potassium carbonate (99\%), and Amberlite MB6113 (for ion chromatography, mixed resin) were purchased from Acros Organics. Glass microscope slides were made in accordance with international standard ISO 8037/1 $(n=1.53 \pm 0.02)$ and were purchased from MLS NV (Menen, Belgium). All materials were used as received unless otherwise stated.

Preparation of a Cellulose Nanocrystal (CNC) Suspension. A cellulose nanocrystal suspension was obtained via sulfuric acid (10.06 M) hydrolysis of cotton wool over $40 \mathrm{~min}$ at $45{ }^{\circ} \mathrm{C}$ using standard literature procedures. $^{20}$ The suspension was then mixed with Amberlite MB6113 resin to remove excess non- $\mathrm{H}^{+}$and $\mathrm{OH}^{-}$ions in solution. The resin was subsequently removed by filtration. Initial aqueous suspensions were concentrated to the desired concentrations by evaporating excess water.
Film Deposition. Glass microscope slides were rinsed with isopropanol and dried with compressed air prior to use. Each film was prepared by depositing $10 \mu \mathrm{L}$ of a suspension of cellulose nanocrystals with a specific concentration under different conditions. The weight concentration of the initial cellulose nanocrystal suspensions was determined with thermogravimetric analysis. Saturated aqueous solutions of $\mathrm{CaCl}_{2}(30 \% \mathrm{RH})$ and $\mathrm{K}_{2} \mathrm{CO}_{3}(40 \%$ $\mathrm{RH})^{21}$ as well as pure ethanol were used in a closed desiccator to obtain environments with a controlled specific relative humidity/ atmosphere.

Cross-Polarized Light Microscope. Cross-polarized light microscope images were taken with an Olympus BX51 microscope. The glass slide was placed between crossed polarizers, and images were recorded with a color digital CCD camera (Lumenera Infinity $2 \mathrm{Mpx}$ ).

Profilometer. Profilometer traces were obtained with a TaylorHobson 120-L Form Talysurf using a diamond tip with a radius of 2.5 $\mu \mathrm{m}$ and a measurement speed of $0.5 \mathrm{~mm} / \mathrm{s}$.

Droplet Imaging. Sessile droplets were monitored with a system adapted from ref 22. A Canon EOS 1200D camera was coupled with a Plano-Convex Lens $(f=50 \mathrm{~mm}$, Thorlabs). Measurements were analyzed with ImageJ and drop shape analysis software. ${ }^{23}$ Drop volumes were obtained by acquiring images over time and fitting the droplet shape with a spherical cap.

Rheological Measurement. Aqueous suspensions of CNCs were analyzed using an AR-G2 stress-controlled rotational rheometer (TA Instruments). Steady shear viscosity versus shear rate curves were obtained for each sample at shear rates between 10 and $100 \mathrm{~s}^{-1}$. For each sample, the time required to reach steady state was determined by a transient test whereas amplitude sweep experiments at a frequency of $10 \mathrm{rad} / \mathrm{s}$ were used to determine the strain for frequency sweep experiments. All of the suspensions were tested at $20^{\circ} \mathrm{C}$ using doublewall concentric cylinder geometry with gap sizes of 380 and $420 \mu \mathrm{m}$. A solvent trap was used to avoid water evaporation.

Pendant Drop Tensiometry. The surface tension of pendant drops containing different concentrations of $\mathrm{CNCs}$ was measured with a CAM200 (KSV NIMA) at room temperature. The measurement precision was determined using the Worthington number, $W_{\mathrm{o}}$, which was calculated to be $\sim 0.8$. $^{24}$

Zeta Potential Measurement. Zeta potential measurements were carried out on a NanoBrook Omni (Brookhaven). A BI-SREL electrode was used for measurements using phase analysis light scattering. ${ }^{25-27}$ Five consecutive measurements were taken for each sample. To determine the zeta potential of CNCs in water-ethanol mixtures, the cellulose nanocrystal suspension in water was mixed with the desired amount of ethanol within the cell. Dielectric constants were taken from ref 28.

\section{RESULTS AND DISCUSSION}

A series of films were prepared by drop casting cellulose nanocrystal suspensions under different atmospheres. The effect of the environment on the deposition patterns of the films and the ensuing optical properties of the produced films were then investigated. Under ambient conditions, nano- 
particles accumulate and stack around the perimeter, giving rise to the so-called coffee-ring effect. ${ }^{29}$ This can clearly be seen from the height profile of the deposited film (Figure 1a). The height difference within these outer deposits, which cause thin film interference as illustrated in Figure $1 \mathrm{~b}$ for a drop-cast film from a 3.7 wt \% CNC suspension, leads to a sequence of colored bands that can be observed under a cross-polarized light microscope. Previously, rainbow colors were observed for the obtained films in the literature by the naked eye due to variations in the chiral nematic pitch distance. ${ }^{30,31}$ Increasing the concentration of the initial suspension leads to an increase in the accumulation and increased stacking of particles around the perimeter (Figure 2).

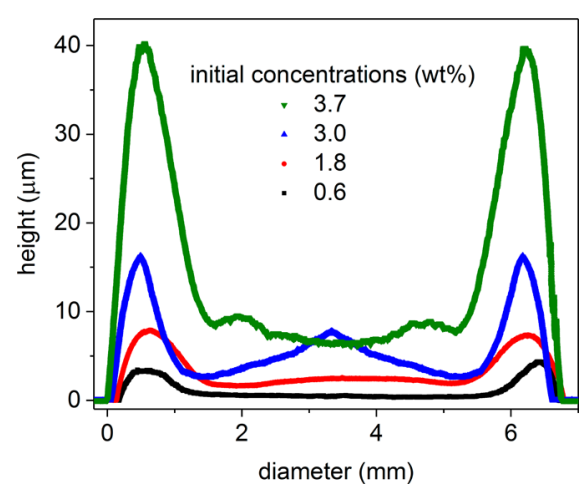

Figure 2. Height profiles of the films obtained by drying droplets with different initial weight concentrations of CNCs.

The rainbow colors at the edge of the film are not visible to the naked eye (Figure 1a) and become visible only under crosspolarized light (Figure $1 \mathrm{~b}$ ). These colors are a direct result of the changing thickness, and the color-thickness relation can be correlated using the Michel-Lévy chart. ${ }^{32}$ The height value obtained using the profilometer results yields a thickness at different positions of the perimeter of the film. We multiplied this value by the refractive index difference of glass and cellulose with $n_{\text {glass }}=1.549$ and $n_{\text {cellulose }}=1.524^{30}$ yielding an optical path difference that fits the colors observed under the cross-polarized light microscope and the colors displayed in the Michel-Lévy chart $^{32}$ (Figure 1b, Figures SI 1-3). Films produced using lower concentrations of CNC suspensions $(0.6,1.8$, and 3 wt \%) did not show any color gradient at the perimeter as the thickness of the coffee ring deposits was not enough to generate color (Figure 2, Figure SI 4).

At the center of the drop-cast films, one observes iridescence both with the naked eye and under cross-polarized light as a result of chiral nematic stacking of the deposited CNCs (Figure $1 \mathrm{a}, \mathrm{c})$. Fingerprints due to chiral nematic structuring are readily observed in some domains in the center (Figure 1c), whereas because of stacking of the nanocrystals at the perimeter it is very difficult to differentiate existing fingerprints at the outer edges. The center of the film in Figure 1 has a uniform average thickness of about $10 \mu \mathrm{m}$, leading to a uniform bluish color that can be explained by $\lambda_{\max }=n P \sin \theta$ using the chiral nematic pitch distance, ${ }^{33}$ where $n$ is the refractive index of the film, $P$ is the helical pitch, and $\theta$ is the incident angle of the light with respect to the surface of the film. However, the pitch length for the blue domains was not measured because of the optical resolution limit of the microscope. ${ }^{34} \lambda_{\max }$ can also be tuned by altering the helical pitch. ${ }^{35}$
The depositions pattern is thus ultimately dependent on the time-dependent concentration in the suspension, and this will continually change during the drying process. Therefore, it becomes crucial to determine the particle concentration in the suspension at any given time during the drying process. Indeed, the anisotropic liquid-crystal phase transition occurs at increased concentrations ( $\sim 3$ wt \% for the CNC particles derived from cotton used in this work and observed tactoid formation inside the capillary, Figure SI 11), whereas the viscosity of the bulk suspension also changes, having a direct effect on particle motion in the suspension. Because we know the volume of the drop during evaporation, the initial concentration of the suspension, the radius change of the drying drop with time from time-dependent measurements, and the mass profile in the deposited film from profilometer measurements, we can determine the average concentration of nanoparticles in the suspension as a function of time during the whole drying process. By applying the chain rule, the change in mass of nanoparticles deposited as a function of time can be expressed as a product of the profilometer profile and the radius change of the evaporating drop:

$$
\frac{\partial m}{\partial t}=\frac{\partial m}{\partial r} \frac{\partial r}{\partial t}
$$

The total area under the profilometer profile $h(r)$ yields $M$, the known initial mass of the solid particles in the drop upon deposition:

$$
\int_{0}^{2 \pi} \int_{0}^{R} h(r) \mathrm{d} r \mathrm{~d} \theta=M
$$

In eq 2 , the profile is assumed in a first approximation to be the same for all cross sections (Figure 2). The validity of this approximation was verified to be acceptable by comparing the height profiles of different cross sections going through the central point.

The change in radius as a function of time $(\mathrm{d} r / \mathrm{d} t)$ is directly determined by time-resolved measurements of the droplet:

$$
\left(\frac{\mathrm{d} r}{\mathrm{~d} t}\right)_{\text {film deposition }}=\left(-\frac{\mathrm{d} r}{\mathrm{~d} t}\right)_{\text {droplet shrinkage }}
$$

Because all contributions can be determined, the deposition rate $\mathrm{d} m / \mathrm{d} t$ can be directly calculated from measured values (Figure SI 5) and normalized with the initial weight concentration of dispersed particles (Figure SI 6). The absolute deposited mass at any given time can then also be calculated by the integration of $\mathrm{d} m / \mathrm{d} t$ and normalization with the initial mass of nanoparticles in the deposited droplet (Figure SI 7).

With this knowledge, it becomes possible to obtain the mass of nanoparticles inside the suspension during drying and, together with the time-resolved evolution of drop volume, to obtain the average concentration of nanoparticles inside the droplet at any time during droplet evaporation (Figure 3). The average concentration of nanoparticles is found to increase continuously during droplet evaporation. The concentration for drops with an initial concentration of $1.8 \mathrm{wt} \%$ and above reaches values well above the percolation threshold where gelation and chiral nematic stacking become possible.

As the concentration of nanoparticles in the droplets increases, nanoparticles will accumulate at the liquid-vapor interface, resulting in a decrease in surface tension (Figure 4, Figure SI 8). This results in the formation of a skin at the liquid-air interface. ${ }^{18,36}$ The transport of water to the surface 


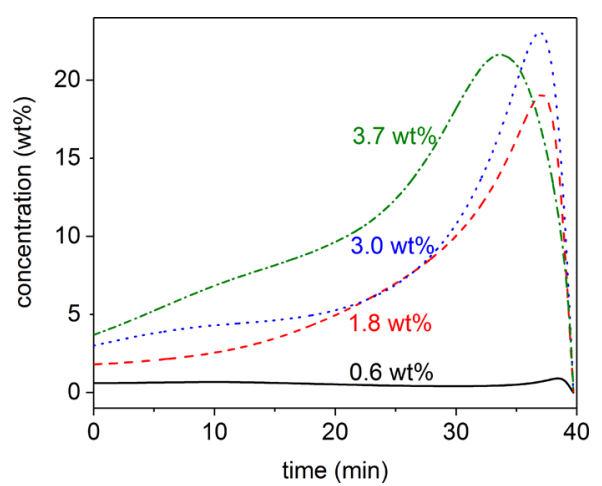

Figure 3. Average weight concentration variation of cellulose nanocrystals in the drying droplets for varying initial concentrations as a function of time.

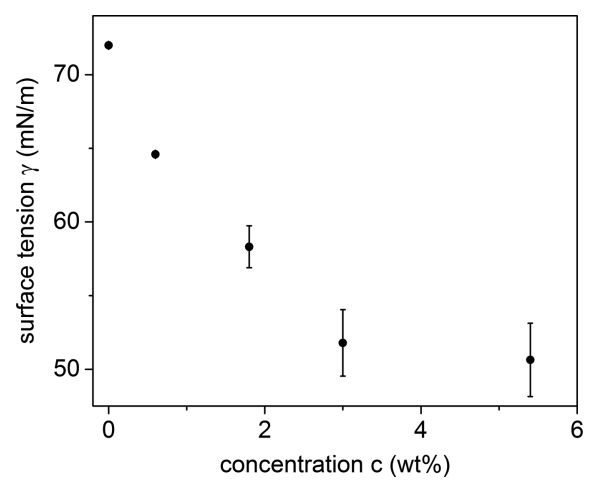

Figure 4. Surface tension measurement of the cellulose nanocrystal suspensions.

thus becomes rate-limited, leading to a decrease in the drying rate at longer evaporation times. Consequently, the weight concentration of the particles at longer evaporation times decreases (Figure 3) as the skin deposits and the suspension becomes richer in water (Figure SI 9). Before skin formation, the suspension becomes highly concentrated during evaporation (Figure 3), but this does not cause rainbow color formation in the center of the obtained films under a crosspolarized light microscope (Figure 1c). It was reported that in the absence of gelation, the pitch distance decreases as the concentration of cellulose nanocrystals in the suspension increases. $^{31}$ This explains the color changes observed under visible light in Figure 1a. However, the symmetry observed in the colored bands at the perimeter under cross-polarized light was a consequence of the symmetric thickness variation of the outer edge deposits as shown in Figure $1 \mathrm{~b}$ and Figure SI 2.

Shortly after the deposition of the drop onto the glass slide, the deposition rate was found to increase for all concentrations (Figure SI 5). For 0.6 and $3.7 \mathrm{wt} \%$, these rates reached a plateau at longer times, which resulted in the formation of a uniform flat deposit in the center of the drop-cast film. On the other hand, $\mathrm{d} m / \mathrm{d} t$ increased for the $3 \mathrm{wt} \%$ starting concentration droplet at longer drying times, giving rise to deposits displaying a central peak (Figure 2). Normalizing these results with respect to the initial weight concentration shows these differences even more clearly (Figure SI 6). The formation of central peaks in films with intermittent starting concentrations can be caused by particle accumulation at the air-water surface, which will create an inward flow during depinning of the drying droplet, ${ }^{37}$ by skin formation, ${ }^{38,39}$ or as a result of the contact line dragging particles with it, pulling them toward the center of the droplet. ${ }^{40}$ It was also shown that similar central deposition peaks could be the result of sol-gel transitions during evaporation. ${ }^{41}$

To understand the effect of surface tension changes, Marangoni numbers, $M_{a}$ (eq 4), were calculated using the concentration changes of the solute and the surface tension to determine the relative magnitude of Marangoni versus convective effects during droplet drying. ${ }^{37,42,43}$

$$
M_{\mathrm{a}}=-\frac{\mathrm{d} \gamma}{\mathrm{d} c} \frac{l_{\mathrm{c}}}{\eta D_{\mathrm{p}}} \Delta c
$$

$M_{\mathrm{a}}$ (Table 1) is based on the change in surface tension $(\gamma)$ with the change in concentration $(c)$, the first derivative of $\gamma(c)$, by

Table 1. Relative Marangoni Numbers for the Cellulose Nanocrystal Suspensions

weight concentration (wt \%)
0.6
1.8
3
3.7

Marangoni numbers 5.57

2.22

1

0.58

fitting an exponential function to Figure 4, the viscosity of the suspension $(\eta)$ at a shear rate of $10 \mathrm{~s}^{-1}$ (Figure SI 10), the length scale of the droplet $\left(l_{\mathcal{c}}\right.$ diameter of the droplet), the particle diffusivity $\left(D_{\mathrm{p}}, 5 \times 10^{-12} \mathrm{~m}^{2} / \mathrm{s}\right),{ }^{44}$ and the concentration difference $(\Delta c)$ between the edge and the top of the droplet approximated by the difference in initial weight concentration and weight concentration during evaporation.

A high $M_{\mathrm{a}}$ is seen for low-weight-concentration dispersions, which would result in a large reverse flow of particles toward the inside of the drying droplet. As a result, the deposit would become more homogeneous. This is indeed seen for $0.6 \mathrm{wt} \%$, which shows a relatively homogeneous deposit without a clear coffee-ring band at the outer edge (Figure 2). As $M_{\mathrm{a}}$ decreases, the contribution of the convective flow of particles toward the outer edge becomes more and more important, and a larger fraction of particles is deposited in the outer ring (Figure 2), leading to enough deposit height to give a Bragg reflection and colored bands for $3.7 \mathrm{wt} \%$ (Figure 1).

Next, we investigated the effect of relative humidity on dropcast films to determine its effect on the deposition patterns. It was expected that controlling the evaporation time by changing the relative humidity would have an effect on the deposition pattern while we measured how the relative humidity affects the evaporation rate. However, the variations in the evaporation rate did not have a direct effect on the obtained ring deposition pattern (Figure.SI 13). This was also observed for the dried nanofluid deposition patterns, which were deposited under different relative humidities. ${ }^{45}$

Understanding the effect of Marangoni flow for suspensions with different starting weight concentrations (Table 1) and the effect of relative humidity on deposition patterns showed that a uniform thickness deposit was not feasible for highly concentrated suspensions. Marangoni flow is only effective at low concentrations ( $0.6 \mathrm{wt} \%)$ to obtain uniform deposits. However, at these low concentrations, the thickness of the deposits was not enough to generate color bands at the perimeter as described previously (Figure SI 4), and relative humidity had only a minor effect on the obtained deposition pattern (Figure SI 13). Thus, to obtain films with a uniform 
deposition height and iridescence, we need to amplify the Marangoni flow for higher concentrations. For this reason, further investigations were carried out on drying films under an ethanol atmosphere. Under ambient conditions, a surface tension gradient is created by changes in the concentration of nanoparticles in the suspension. These surface tension gradients will induce a shear stress at the interface, which will lead to Marangoni flow (eq 5). The evaporation-related coffeering effect can be suppressed by creating a surface tension gradient created via the diffusion of ethanol vapor into the droplet with time, which will create a stronger Marangoni flow dragging particles back to the center of the droplet and thereby helping to suppress the accumulation of particles at the edge.

$$
\begin{aligned}
& \sigma_{\text {outside }}-\sigma_{\text {inside }}=\vec{t} \cdot \vec{\nabla} \gamma_{\text {lv }} \\
& \sigma=\eta\left(\frac{\partial u_{r}}{\partial z}+\frac{\partial u_{z}}{\partial r}\right)
\end{aligned}
$$

Combining equations of shear stress and Marangoni flow (eqs 5 and 6), ${ }^{46}$ the dynamic boundary condition for Marangoni flows is obtained as (eq 7$)^{47,48}$

$$
\eta_{1} \frac{\partial u_{t 1}}{\partial n}+\eta_{1} \frac{\partial u_{n 1}}{\partial t}-\eta_{2} \frac{\partial u_{t 2}}{\partial n}-\eta_{2} \frac{\partial u_{n 2}}{\partial t}=-[\nabla \gamma-\hat{n}(\hat{n} \cdot \nabla \gamma)]
$$

where the right-hand side of eq 7 describes the tangential stress caused by surface tension, $\sigma_{\text {inside, }} \sigma_{\text {outside }}$ are the tangential stresses in the inner and outer domains of the droplet interface, respectively, $\nabla \gamma$ is the surface tension gradient, $\eta$ is the viscosity of the medium, $u_{\mathrm{r}}$ is the radial velocity, $u_{\mathrm{t}}$ is the velocity tangent to the interface, $u_{\mathrm{n}}$ is the normal component, and $\hat{n}$ is the unit normal pointing out of the surface.

The modified Péclet number $\left(P e_{M_{\mathrm{a}}}\right)$ (eq 8) was used to compare the magnitude of the Marangoni force to the viscous forces and the diffusivity, whereas $P e_{\mathrm{E}}$ was used to compare the evaporative flux, which is responsible for the capillary flow and thus the ring deposition pattern, to the diffusivity. ${ }^{49}$ The ratio of two numbers, $P e_{M_{a} / E}$, results in a relationship of the Marangoni force to the capillary flow

$$
P e_{M_{\mathrm{a}}}=\frac{\Delta \gamma l_{\mathrm{c}}}{\eta D_{\mathrm{p}}} \quad P e_{E}=\frac{v_{\mathrm{c}} l_{\mathrm{c}}}{D_{\mathrm{p}}} \quad P e_{M_{\mathrm{a}} / E}=\frac{\Delta \gamma}{\eta v_{\mathrm{c}}}
$$

where $\Delta \gamma$ is the surface tension change and can be estimated to be $\sim 27 \mathrm{mN} / \mathrm{m}$ for a $3 \mathrm{wt} \%$ suspension dried under pure ethanol in which $\gamma_{3 \mathrm{wt}} \%$ droplet was determined experimentally (Figure 4), $\gamma_{\text {ethanol }}$ is $22 \mathrm{mN} / \mathrm{m},{ }^{50} v_{\mathrm{c}} \approx J_{\mathrm{e}} / l_{\mathrm{c}}^{2}$ is the evaporation velocity, $J$ is the evaporation flux (Figure SI 9), $D_{\mathrm{p}}$ is the particle diffusivity, $l_{c}$ is the length scale of the droplet (diameter of the droplet), and $\eta$ is the viscosity of the suspension (Figure SI 10).

$P e_{M_{\mathrm{a}} / E}$ can thus be calculated to be $4.7 \times 10^{7}$ for the suspension drying under ethanol, meaning that Marangoni flow is dominant over capillary flow and viscous forces under an ethanol atmosphere.

On the other hand, the colloidal stability is critical when we aim to control the flow of the particles in the suspension, and it has a direct effect on the optical properties of obtained films. For this, the colloidal stability of CNC suspensions in binary water-ethanol mixtures was characterized by rheological and zeta potential measurements. Atmospheric ethanol will diffuse into the droplet, where at high-enough concentration this may induce the collapse of the double layer and cause the aggregation of cellulose nanocrystals. For example, for a 0.6 wt $\%$ cellulose suspension in water this is seen as the zeta potential decreased from $-47 \mathrm{mV}$ in pure water to $-20 \mathrm{mV}$ $(3: 1 \mathrm{v} / \mathrm{v}$ water-ethanol) and $-12 \mathrm{mV}(1: 1 \mathrm{v} / \mathrm{v}$ water-ethanol) upon addition of ethanol to the suspension. For $3.7 \mathrm{wt} \%$ in water, the zeta potential decreased from -34 to $-18 \mathrm{mV}$ (3:1 $\mathrm{v} / \mathrm{v}$ water-ethanol) and $-14 \mathrm{mV}(1: 1 \mathrm{v} / \mathrm{v}$ water-ethanol). The flocculation of CNCs in the suspension was also observed with the naked eye upon addition of excess ethanol.

Rheological measurements showed an increase in viscosity with an increasing amount of ethanol added to the suspension (Figure 5a, Figure SI 14), an indication of aggregation in these
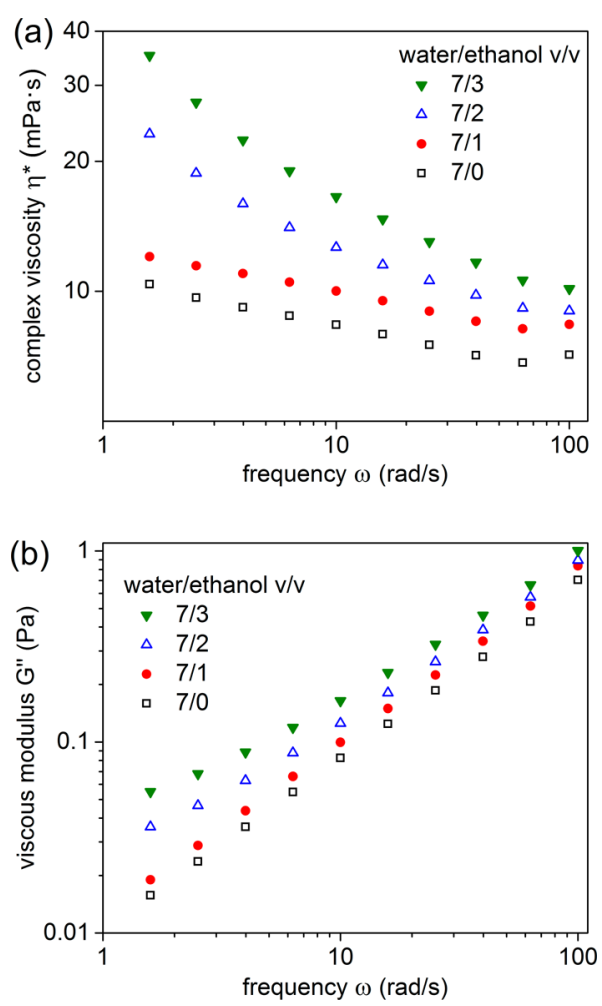

Figure 5. Dynamic oscillatory measurements: (a) complex viscosity and (b) viscous modulus of initial $3 \mathrm{wt} \% \mathrm{CNC}$ suspensions in water/ ethanol (v/v).

systems. The coupled hydrodynamic effects caused an increase in the viscous modulus at higher frequencies (Figure 5b). The viscous modulus was also found to dominate the elastic modulus for all cases, so there is no observed viscoelastic behavior or gelation (Figure SI 15).

After characterizing the flow and the colloidal stability of the CNC particles, the optical properties of films obtained under an ethanol atmosphere were investigated. The drying of aqueous CNC suspension droplets shows a more homogeneous particle deposition for the films formed under ethanol as the result of a much stronger Marangoni flow. However, the cross-polarized light microscope images showed no evidence of color formation in the dried film (Figure SI 16). Films obtained by drying different weight percentages of CNC suspensions under ethanol vapor have a white color when viewed with the naked eye. This may indicate that the colloidal stability of the CNCs was too negatively affected by ethanol diffusion into the drying droplet to form the structured patterning needed for iridescence. We therefore decided to reduce the amount of 
ethanol in the atmosphere, and the same experiment was carried out under an atmosphere created by letting a mixture of 60:40 v/v ethanol-water equilibrate within an enclosed environment. $P e_{M_{a} / E}$ is then reduced to $3.8 \times 10^{7}$ for a $3 \mathrm{wt}$ $\%$ suspension in water drying under a $60: 40 \mathrm{v} / \mathrm{v}$ ethanol-water atmosphere $\left(\gamma_{60: 40 \text { ethanol/water }}=27 \mathrm{mN} / \mathrm{m}\right),{ }^{50}$ which is adequate to obtain sufficient Marangoni flow. As a result, a smooth deposit (Figure $6 \mathrm{~b}$ ) without the aggregation of nanoparticles

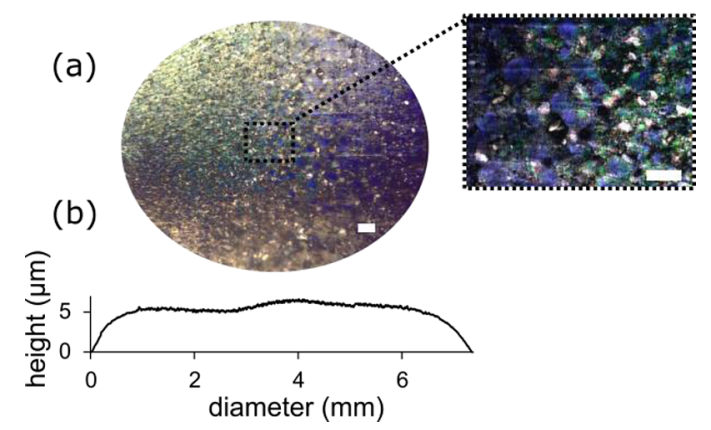

Figure 6. (a) Cross-polarized light microscope images of the film obtained by drying a $3 \mathrm{wt} \% \mathrm{CNC}$ suspension under vapor obtained by a mixture of $60: 40 \mathrm{v} / \mathrm{v}$ ethanol-water (scale bars $100 \mu \mathrm{m}$ ). (b) Height profile of the film versus diameter.

was obtained with a starting $3 \mathrm{wt} \% \mathrm{CNC}$ suspension, leading to bluish uniform iridescent color formation throughout the film (Figure 6a). Control of deposition was not feasible for lessconcentrated suspensions because of the high spreading of the suspension at initial drying times.

\section{CONCLUSIONS}

Iridescent films with a uniform deposition of cellulose nanocrystals were obtained by controlling the relative magnitude of the Marangoni flow. The influencing forces in the deposition process were successfully described using the Marangoni number, which enabled us to describe the studied systems and develop a framework to control the deposition profile. This can readily be applied to different atmospheres and droplet compositions.

The mass transfer of the suspended particles investigated in different environments and the deposition rate were expressed with combined experiments of the height measurements of the films and droplet imaging in real time for the drying suspension. The colloidal stability was characterized by rheological and zeta potential measurements to optimize the optical properties of the obtained films through the desired structure formation.

\section{ASSOCIATED CONTENT}

\section{S Supporting Information}

The Supporting Information is available free of charge on the ACS Publications website at DOI: 10.1021/acs.langmuir.6b03724.

More detailed analysis of cross-polarized light microscope images, an analysis of the deposition process, and rheological measurements (PDF)

Film deposition of the drying of a droplet of a suspension of $3.7 \mathrm{wt} \%$ cellulose nanocrystals viewed under a crosspolarized light microscope, showing the formation of colored bands as more particles accumulate at the outer edge, resulting in a rainbowlike coffee ring $\mathrm{MPG}$

\section{AUTHOR INFORMATION}

\section{Corresponding Author}

*E-mail: wim.thielemans@kuleuven.be. Phone: +32 (0)56 24 6171.

ORCID

Christina Schütz: 0000-0003-0238-1639

Wim Thielemans: 0000-0003-4451-1964

\section{Present Address}

Renewable Materials and Nanotechnology Research Group, Department of Chemical Engineering, KU Leuven, Campus Kulak Kortrijk, Etienne Sabbelaan 53, 8500 Kortrijk, Belgium

\section{Author Contributions}

A.G. and W.T. developed the concept for the study. A.G. performed the experiments, processed the data, and wrote the manuscript. A.G., C.S., and W.T. interpreted the data and reviewed the manuscript.

Notes

The authors declare no competing financial interest.

\section{ACKNOWLEDGMENTS}

The authors thank Research Foundation-Flanders (FWO) for funding under the Odysseus grant (G.0C60.13N) and KU Leuven for grant OT/14/072. W.T. also thanks the Provincie West-Vlaanderen (Belgium) for financial support through his Provincial Chair in Advanced Materials.

\section{REFERENCES}

(1) Lagerwall, J. P. F.; Schütz, C.; Salajkova, M.; Noh, J.; Hyun Park, J.; Scalia, G.; Bergström, L. Cellulose nanocrystal-based materials: from liquid crystal self-assembly and glass formation to multifunctional thin films. NPG Asia Mater. 2014, 6, e80.

(2) Deegan, R. D. Pattern formation in drying drops. Phys. Rev. E: Stat. Phys., Plasmas, Fluids, Relat. Interdiscip. Top. 2000, 61 (1), 475485.

(3) $\mathrm{Mu}, \mathrm{X}$.; Gray, D. Droplets of cellulose nanocrystal suspensions on drying give iridescent 3-D "coffee-stain" rings. Cellulose 2015, 22 (2), 1103-1107.

(4) Uhlig, M.; Fall, A.; Wellert, S.; Lehmann, M.; Prévost, S.; Wågberg, L.; von Klitzing, R.; Nyström, G. Two-Dimensional Aggregation and Semidilute Ordering in Cellulose Nanocrystals. Langmuir 2016, 32 (2), 442-450.

(5) Wang, P.-X.; Hamad, W. Y.; MacLachlan, M. J. Structure and transformation of tactoids in cellulose nanocrystal suspensions. Nat. Commun. 2016, 7, 11515.

(6) Nyström, G.; Fall, A. B.; Carlsson, L.; Wågberg, L. Aligned cellulose nanocrystals and directed nanoscale deposition of colloidal spheres. Cellulose 2014, 21 (3), 1591-1599.

(7) Maren, R; Fernando, N., Deposition of Cellulose Nanocrystals by Inkjet Printing. In Model Cellulosic Surfaces; American Chemical Society: 2009; Vol. 1019, pp 157-171.

(8) Sun, J.; Bao, B.; He, M.; Zhou, H.; Song, Y. Recent Advances in Controlling the Depositing Morphologies of Inkjet Droplets. ACS Appl. Mater. Interfaces 2015, 7 (51), 28086-28099.

(9) Yakovlev, A. V.; Milichko, V. A.; Vinogradov, V. V.; Vinogradov, A. V. Inkjet Color Printing by Interference Nanostructures. ACS Nano 2016, 10 (3), 3078-3086.

(10) Wu, L.; Dong, Z.; Li, F.; Zhou, H.; Song, Y. Emerging Progress of Inkjet Technology in Printing Optical Materials. Adv. Opt. Mater. 2016, DOI: 10.1002 /adom.201600466.

(11) Moon, R. J.; Martini, A.; Nairn, J.; Simonsen, J.; Youngblood, J. Cellulose nanomaterials review: structure, properties and nanocomposites. Chem. Soc. Rev. 2011, 40 (7), 3941-3994.

(12) Bardet, R.; Belgacem, N.; Bras, J. Flexibility and Color Monitoring of Cellulose Nanocrystal Iridescent Solid Films Using 
Anionic or Neutral Polymers. ACS Appl. Mater. Interfaces 2015, 7 (7), 4010-4018.

(13) Wang, B.; Walther, A. Self-Assembled, Iridescent, CrustaceanMimetic Nanocomposites with Tailored Periodicity and Layered Cuticular Structure. ACS Nano 2015, 9 (11), 10637-10646.

(14) Guidetti, G.; Atifi, S.; Vignolini, S.; Hamad, W. Y. Flexible Photonic Cellulose Nanocrystal Films. Adv. Mater. 2016, 28 (45), 10042-10047.

(15) Viet, D.; Beck-Candanedo, S.; Gray, D. Dispersion of cellulose nanocrystals in polar organic solvents. Cellulose 2007, 14 (2), 109113.

(16) Cheung, C. C. Y.; Giese, M.; Kelly, J. A.; Hamad, W. Y.; MacLachlan, M. J. Iridescent Chiral Nematic Cellulose Nanocrystal/ Polymer Composites Assembled in Organic Solvents. ACS Macro Lett. 2013, 2 (11), 1016-1020.

(17) Zhang, Y.; Yang, S.; Chen, L.; Evans, J. R. G. Shape Changes during the Drying of Droplets of Suspensions. Langmuir 2008, 24 (8), $3752-3758$.

(18) Jativa, F.; Schütz, C.; Bergström, L.; Zhang, X.; Wicklein, B. Confined self-assembly of cellulose nanocrystals in a shrinking droplet. Soft Matter 2015, 11 (26), 5374-5380.

(19) Uetani, K.; Yano, H. Self-organizing capacity of nanocelluloses via droplet evaporation. Soft Matter 2013, 9 (12), 3396-3401.

(20) Jasmani, L.; Eyley, S.; Wallbridge, R.; Thielemans, W. A facile one-pot route to cationic cellulose nanocrystals. Nanoscale 2013, 5 (21), 10207-10211.

(21) Winston, P. W.; Bates, D. H. Saturated Solutions For the Control of Humidity in Biological Research. Ecology 1960, 41 (1), 232-237.

(22) Lamour, G.; Hamraoui, A.; Buvailo, A.; Xing, Y.; Keuleyan, S.; Prakash, V.; Eftekhari-Bafrooei, A.; Borguet, E. Contact Angle Measurements Using a Simplified Experimental Setup. J. Chem. Educ. 2010, 87 (12), 1403-1407.

(23) Stalder, A. F.; Kulik, G.; Sage, D.; Barbieri, L.; Hoffmann, P. A snake-based approach to accurate determination of both contact points and contact angles. Colloids Surf., A 2006, 286 (1-3), 92-103.

(24) Berry, J. D.; Neeson, M. J.; Dagastine, R. R.; Chan, D. Y. C.; Tabor, R. F. Measurement of surface and interfacial tension using pendant drop tensiometry. J. Colloid Interface Sci. 2015, 454, 226-237.

(25) Clogston, J.; Patri, A., Zeta Potential Measurement. In Characterization of Nanoparticles Intended for Drug Delivery, McNeil, S. E., Ed.; Humana Press: 2011; Vol. 697, pp 63-70.

(26) Tscharnuter, W. W. Mobility measurements by phase analysis. Appl. Opt. 2001, 40 (24), 3995-4003.

(27) Prathapan, R.; Thapa, R.; Garnier, G.; Tabor, R. F. Modulating the zeta potential of cellulose nanocrystals using salts and surfactants. Colloids Surf., A 2016, 509, 11-18.

(28) Wyman, J. The dielectric constant of mixtures of ethyl alcohol and water from $-5^{\circ} \mathrm{C}$ to $40^{\circ} \mathrm{C}$. J. Am. Chem. Soc. 1931, 53 (9), 32923301.

(29) Deegan, R. D.; Bakajin, O.; Dupont, T. F.; Huber, G.; Nagel, S. R.; Witten, T. A. Capillary flow as the cause of ring stains from dried liquid drops. Nature 1997, 389 (6653), 827-829.

(30) Dumanli, A. G.; van der Kooij, H. M.; Kamita, G.; Reisner, E.; Baumberg, J. J.; Steiner, U.; Vignolini, S. Digital Color in Cellulose Nanocrystal Films. ACS Appl. Mater. Interfaces 2014, 6 (15), 1230212306.

(31) Mu, X.; Gray, D. G. Formation of Chiral Nematic Films from Cellulose Nanocrystal Suspensions Is a Two-Stage Process. Langmuir 2014, 30 (31), 9256-9260.

(32) Stoiber, R. E.; Morse, S. A. Crystal Identification with the Polarizing Microscope; Chapman \& Hall: New York, 2012.

(33) de Bries, Hl. Rotatory power and other optical properties of certain liquid crystals. Acta Crystallogr. 1951, 4 (3), 219-226.

(34) Parker, R. M.; Frka-Petesic, B.; Guidetti, G.; Kamita, G.; Consani, G.; Abell, C.; Vignolini, S. Hierarchical Self-Assembly of Cellulose Nanocrystals in a Confined Geometry. ACS Nano 2016, 10 (9), 8443-8449.
(35) Shopsowitz, K. E.; Qi, H.; Hamad, W. Y.; MacLachlan, M. J. Free-standing mesoporous silica films with tunable chiral nematic structures. Nature 2010, 468 (7322), 422-425.

(36) Pauchard, L.; Allain, C. Mechanical instability induced by complex liquid desiccation. C. R. Phys. 2003, 4 (2), 231-239.

(37) $\mathrm{Hu}, \mathrm{H}$.; Larson, R. G. Analysis of the Effects of Marangoni Stresses on the Microflow in an Evaporating Sessile Droplet. Langmuir 2005, 21 (9), 3972-3980.

(38) Anyfantakis, M.; Geng, Z.; Morel, M.; Rudiuk, S.; Baigl, D. Modulation of the Coffee-Ring Effect in Particle/Surfactant Mixtures: the Importance of Particle-Interface Interactions. Langmuir 2015, 31 (14), 4113-4120.

(39) Varanakkottu, S. N.; Anyfantakis, M.; Morel, M.; Rudiuk, S.; Baigl, D. Light-Directed Particle Patterning by Evaporative Optical Marangoni Assembly. Nano Lett. 2016, 16 (1), 644-650.

(40) Larson, R. G. Transport and deposition patterns in drying sessile droplets. AIChE J. 2014, 60 (5), 1538-1571.

(41) Talbot, E. L.; Yang, L.; Berson, A.; Bain, C. D. Control of the Particle Distribution in Inkjet Printing through an Evaporation-Driven Sol-Gel Transition. ACS Appl. Mater. Interfaces 2014, 6 (12), 95729583.

(42) Ouenzerfi, S.; Harmand, S. Experimental Droplet Study of Inverted Marangoni Effect of a Binary Liquid Mixture on a Nonuniform Heated Substrate. Langmuir 2016, 32 (10), 2378-2388.

(43) Sempels, W.; De Dier, R.; Mizuno, H.; Hofkens, J.; Vermant, J. Auto-production of biosurfactants reverses the coffee ring effect in a bacterial system. Nat. Commun. 2013, 4, 1757.

(44) Boluk, Y.; Danumah, C. Analysis of cellulose nanocrystal rod lengths by dynamic light scattering and electron microscopy. J. Nanopart. Res. 2014, 16 (1), 1-7.

(45) Brutin, D. Influence of relative humidity and nano-particle concentration on pattern formation and evaporation rate of pinned drying drops of nanofluids. Colloids Surf., A 2013, 429, 112-120.

(46) Plawsky, J. L. Transport Phenomena Fundamentals, 3rd ed.; CRC Press: 2014

(47) Kirby, B. J. Micro- and Nanoscale Fluid Mechanics: Transport in Microfluidic Devices; Cambridge University Press: 2010.

(48) Leal, L. G. Advanced Transport Phenomena: Fluid Mechanics and Convective Transport Processes; Cambridge University Press: 2007.

(49) Majumder, M.; Rendall, C. S.; Eukel, J. A.; Wang, J. Y. L.; Behabtu, N.; Pint, C. L.; Liu, T.-Y.; Orbaek, A. W.; Mirri, F.; Nam, J.; Barron, A. R.; Hauge, R. H.; Schmidt, H. K.; Pasquali, M. Overcoming the "Coffee-Stain" Effect by Compositional Marangoni-Flow-Assisted Drop-Drying. J. Phys. Chem. B 2012, 116 (22), 6536-6542.

(50) Vazquez, G.; Alvarez, E.; Navaza, J. M. Surface Tension of Alcohol Water + Water from 20 to 50.degree.C. J. Chem. Eng. Data 1995, 40 (3), 611-614. 